\title{
Trabajos en Proyectos como Herramienta para Fortalecer las Habilidades Cognitivas en los Estudiantes
}

\author{
Alma A. Benítez ${ }^{(1)}$ y Martha L. García ${ }^{(2)}$ \\ (1) Instituto Politécnico Nacional, CECyT 11. Wilfrido Massieu, Av. de los Maestros 217, \\ CP 11340 Col. Casco de Santo Tomás. México D. F.-México (e-mail: abenitez@ipn.mx) \\ (2) Instituto Politécnico Nacional, ESIME-ZACATENCO, Av. Instituto Politécnico Nacional S/N, \\ Col. Zacatenco, Deleg. Gustavo A. Madero D.F.-México (e-mail: martha.garcia@gmail.com)
}

Recibido Ene. 06, 2011; Aceptado Feb. 17, 2011; Versión final recibida Mar. 02, 2011

\begin{abstract}
Resumen
Se presenta la implementación de un modelo educativo centrado en el aprendizaje del estudiante, realizado en el Instituto Politécnico Nacional (IPN) en México. El modelo se enmarca en el Proyecto Aula, en el que se propone desarrollar una metodología de trabajo a través de la solución de un problema definido en un proyecto. En el CECyT 11 Wilfrido Massieu, se ha implementado ésta forma de trabajo, con alumnos de tercer semestre en un proyecto denominado Robot Luchador. El presente trabajo identifica y analiza las habilidades cognitivas que el alumno desarrolla cuando ha tenido la vivencia de observar, identificar, interpretar y explorar un problema real a través de un proyecto. Para el análisis de los resultados se llevó a cabo un estudio de caso que permitió reconocer el progreso del grupo. Se muestra que la metodología permite fortalecer las habilidades cognitivas del estudiante, tales como interpretar, analizar sintetizar, deducir y razonar.
\end{abstract}

\section{Working in Projects as a Tool to Strengthen Cognitive Skills in Students}

\begin{abstract}
The implementation of an educational model focused on student learning in the National Polytechnic Institute (IPN) in México is presented. The model is developed within the Classroom Project, in which the methodology consists of solving a problem defined in a project. Particularly in the CEСУT 11 Wilfrido Massieu, this form of working with students of the third semester has been implemented in a project called Fighter Robot. This study identifies and analyzes the cognitive skills that students develop when they have the experience of observing, identifying, interpreting and exploring a real problem within a major project. For the analysis of the results a case study was analyzed. The case helped identifying the progress of the group. It is shown that the methodology allows strengthening the cognitive skills of the students, such as interpretation, analysis, synthesis, deduction and reasoning.
\end{abstract}




\section{INTRODUCCIÓN}

Los proyectos se han convertido en una vía prometedora para elevar los aprendizajes de los estudiantes, en todas las áreas de conocimiento, reconociendo su importancia como estrategia metodológica para el desarrollo de capacidades y habilidades necesarias en el mundo actual, impulsando la solución de problemas reales (Abrantes, 1994). El trabajo de los estudiantes en proyectos se considera una estrategia potente para la formación de un pensamiento globalizado, donde se asocian relaciones, estructuras, procedimientos, formas de razonamiento, es decir representa la construcción personal, en el sentido del uso del conocimiento, lo que hace el estudiante con lo que conoce (Aravena, 2001). En este sentido, la teoría del aprendizaje significativo de Ausubel, Novak y Hanesian (1983), permite dar la significatividad de aprendizaje como la inserción en prácticas sociales con sentido utilidad y eficiencia, relacionadas con los desempeños de comprensión, que son actuaciones, actividades, tareas y proyectos. Todas estas dimensiones se articulan claramente como un conjunto de conocimientos habilidades, actitudes, comprensiones y disposiciones cognitivas, socio-afectivas y psicomotoras apropiadamente relacionadas entre sí para facilitar el desempeño flexible, eficaz y con sentido de una actividad en contextos relativamente nuevos y retadores, que se caracteriza por la búsqueda de las relaciones que se pueden establecer en torno a un tema. Varios investigadores en otras partes del mundo también han discutido este tipo de técnicas para mejorar el proceso de enseñanza-aprendizaje (Morrison, 2003; Doveston y Keenaghan, 2006; Koretsky et al., 2008).

Por lo que, trabajar problemas a través de proyectos se considera una estrategia potente para la formación del pensamiento globalizado, que se concibe como el proceso para establecer relaciones en un tema o bien a nivel macro, es decir, entre culturas (Ricks, 2010) donde las relaciones se establecen bajo tres dimensiones; alumno, escuela y cultura, adquiriendo las condiciones para las relaciones interculturales e interpersonales (Hendrickson, 2010), factor clave para el trabajo de proyectos. Resulta entonces indispensable la interacción en dos dimensiones a nivel aula y a nivel escuela, ya que permite relacionar el conocimiento y su campo de aplicación, vincular ideas de otras áreas para lograr nuevas visiones, asociar necesidades y recursos, así como resolver problemas en un medio multicultural (Ricks, 2010). Otra de las bondades que ofrecen los proyectos es que ayuda a fortalecer la creatividad, en el sentido de la flexibilidad para afrontar diversas tareas, la autonomía, la toma de decisiones, la capacidad de síntesis y la organización de pensamiento flexible frente situaciones nuevas haciendo uso de sus fortalezas individuales de aprendizaje y de sus diferentes enfoques hacia este (Thomas, 1998).

La metodología basada en proyectos se puede implementar para el aprendizaje en cualquier área de conocimiento, en particular resulta conveniente para el aprendizaje de la matemática, debido a que el alumno tiene la posibilidad de matematizar el mundo real, lo que implica interpretar datos; establecer relaciones y conexiones; poner en juego conceptos; analizar regularidades; establecer patrones de cambio; encontrar, elaborar, diseñar y/o construir modelos. Cuando se habla de la actividad matemática en la escuela, se destaca que el alumno aprende matemáticas "haciendo matemáticas", lo que supone como esencial la resolución de problemas de la vida diaria, esto implica que desde el principio se integren al currículo una variedad de problemas relacionados con el entorno de los estudiantes. La resolución de problemas en un amplio sentido se considera siempre en conexión con las aplicaciones y la modelación. La forma de describir ese juego o interrelación entre el mundo real y las matemáticas es la modelización. Niss (1989) entiende por modelización el arte de aplicar las matemáticas a la vida real. Gómez (2003), basándose en la definición de Niss, considera que un modelo matemático es una terna donde participan; Objetos del mundo real; conjunto de expresiones matemáticas y, la relación entre ambas. Lesh y Lehrer (2003) mencionan que la modelización matemática es el proceso por el cual se interpreta y se describe matemáticamente situaciones para tomar algún tipo de decisión, lo cual implica centrarse en elementos de la situación, sus relaciones, patrones y características, mientras que "modelo" lo definen como el producto de una descripción matemática de una situación que se construye para describirla, pensar sobre ella, explicarla o realizar predicciones sobre su comportamiento y, por tanto, debe centrarse en las características estructurales de esta situación. 
En el CECyT 11 "Wilfrido Massieu" del Nivel Medio Superior (NMS) se ha implementado la metodología basada en proyectos, considerando las etapas que marcan su desarrollo. En particular el proyecto denominado "Robot Luchador" fue diseñado y construido por alumnos de tercer semestre (15 a 16 años) de la carrera de electrónica. Este documento analiza y expone la identificación de las habilidades cognitivas que el alumno desarrolla cuando explora el contenido de múltiples representaciones durante el proceso de construcción de un modelo matemático en el desarrollo de un proyecto.

\section{MARCO TEÓRICO}

Diversas han sido las investigaciones para analizar el desempeño que tienen las representaciones en el aprendizaje de la matemática, así como estudiar la influencia que posee el manejo de varias representaciones para lograr la aprehensión del objeto matemático. En esta dirección el trabajo desarrollado por Janvier (1987) enfatiza la importancia de las representaciones, pues considera que ellas son elementos fundamentales para establecer comunicación con el medio que le rodea, pero además concibe la idea de explorar significaciones entre representaciones o modos de representación a través del "proceso de traducción", el cual es básico en la adquisición de un objeto. Los trabajos desarrollados por Kaput (1991), Goldin \& Kaput (1996) y Duval (2002), respectivamente han destacado con mayor precisión el papel que desempeña emplear varios sistemas de representación en el proceso de la adquisición de un concepto, aunque sus posiciones no coincidan en su totalidad.

Kaput (1991) basa su análisis en la tradicional estructura del signo lingüístico tradicional, pero además amplia ésta posición, ya que establece la relación entre lo que él llama notación A y su referente $\mathrm{B}, \mathrm{y}$ aclara que todo puede ser expresado en forma material e incluso su correspondencia (posiblemente), pero como menciona, dicha relación se establece a nivel mental. La relación referencial como menciona Kaput, es semánticamente significativa, si los caracteres externos que constituyen a la gráfica son manejables en sus representaciones internas, caso en el cual la ecuación puede ser pensada como el representante de la gráfica. De esta forma las representaciones internas y sus relaciones semánticas, deben tener cierto nivel de desarrollo necesario para que las representaciones externas puedan ser significativamente interpretadas por el individuo. La propuesta que sustenta Kaput \& Goldin (1996) se orienta a las transformaciones de una representación a otra, a un nivel mental, actividad que adquiere un papel decisivo en la construcción de conceptos matemáticos. Sin embargo, Duval (2002) considera que las condiciones cognitivas internas de un sujeto para lograr la aprehensión del concepto, se enfocan en el desarrollo y fortalecimiento de "La Arquitectura Cognitiva", a través de una organización sólida de diferentes sistemas semióticos. Esta actividad se logra cuando los sistemas de representación semióticas, adquieren el rango de registros semiótico, pues se habla de los sistemas de producción necesarios en toda representación, y de las transformaciones que pueden tener, tratamiento y conversión.

Duval plantea la siguiente pregunta: “¿Cuáles son las condiciones cognitivas internas requeridas para que cualquier estudiante pueda entender matemáticas?", (Duval, 2002 p. 320), la cual cuestiona las condiciones cognitivas para que el estudiante pueda resolver tareas que requieran el empleo de al menos dos representaciones, es decir la coordinación de varios sistemas semióticos, es una actividad cognitiva que es fundamental para el entendimiento de la matemática, pues requiere su organización. Bajo estas condiciones el aprendizaje de la matemática significa integrar en la Arquitectura Cognitiva todos los registros semióticos, así como nuevos sistemas de representación, para su coordinación. Ello implica la necesidad de considerar la actividad cognitiva de Conversión, una tarea fundamental en el proceso para lograr la aprehensión del objeto, y por consecuencia el fortalecimiento de la Arquitectura Cognitiva, lo cual contribuye a crear y desarrollar habilidades en el estudiante para enfrentar nuevos retos en su formación.

Parnafes y DiSessa (2004) indican que la reflexión de los estudiantes esta ligada a la representación y al contexto que emplean, además las autoras consideran que cada representación resalta un aspecto del concepto y que cuando los estudiantes emplean varias representaciones desarrollan un entendimiento más flexible del concepto. Parnafes y DiSessa se 
basaron en la nación del efecto representacional para examinar la idea de que particularmente diferentes representaciones apoyan y fortalecen diferentes procesos cognitiva, en particular, los distintos tipos de razonamiento. Ellas investigaron los procesos cognitivos que los estudiantes emplean en la resolución de problemas de movimiento mediante el uso de un micromundo (llamado "NumberSpeed") que tuvo dos representaciones: la representación del movimiento (animación basada) y la representación número de listas (basado en tablas). Dos tipos distintos de razonamiento son reconocido en el aprendizaje de los alumnos al interactuar con NumberSpeed: (1) basado en el razonamiento de modelos y (2) el razonamiento basado en restricciones, refiriéndose al uso de una variedad de representaciones en dos diferentes caminos; a) el uso de las representaciones para entender con mayor profundidad un concepto y b) la utilización de una representación a fin de promoverlos procesos cognitivos, como la abstracción y generalización.

Particularmente, Benítez (2009) menciona la relevancia que adquieren las representaciones en la resolución de eventos contextualizados, ya que facilita al alumno dar sentido a la información que le brinda el evento contextualizado y opera hasta dar respuesta a la exigencia solicitada, además considera que la primera representación con la cual se inicia el proceso de solución es decisiva, ya que se presenta entre la percepción del problema y el proceso de resolución, durante el cual influyen varios aspectos como son: la formulación del problema, las ideas previas del estudiante, las condiciones dentro de las cuales el problema está inmerso, factores que son determinantes para que el estudiante pueda re-interpretar o modificar la primera representación, cuyo tratamiento conlleva a identificar información para hacer inferencias y seleccionar los elementos relevantes que posteriormente se traducirán en la abstracción del análisis de las partes y su integración, dando lugar a la síntesis y a la conclusión del problema.

\section{METODOLOGÍA}

En el Instituto Politécnico Nacional se ha implementado un modelo educativo centrado en el aprendizaje del estudiante, con el cual se pretende educar a los estudiantes para la vida, es decir aprender a aprender que involucra el saber ser, hacer, coincidir y conocer. Este Modelo Educativo, es un marco de referencia institucional que favorece la formación general de todos los niveles y modalidades, particularmente el Nivel Medio Superior. Este Modelo ha implementado el "proyecto de aula" (Materiales para la Reforma, IPN, 2004) como una alternativa para desarrollar una metodología de trabajo áulico, innovador, colaborativo e interdisciplinario, a través de la solución de un problema, definido en un proyecto, fomentando la enseñanza en el desarrollo de habilidades, actitudes y conocimientos en el alumno, así como el trabajo colaborativo, el fortalecimiento del liderazgo, autoestima, seguridad y convivencia social.

El proyecto tiene como el objetivo: "Desarrollar una nueva cultura de trabajo académico en las aulas, que incorpore procesos centrados en el aprendizaje, que modifique las acciones de intervención del docente, readecue los métodos tradicionales de evaluación y fomente el aprendizaje colaborativo y autónomo". Así, el Proyecto Aula contempla los fines institucionales, pero más aún la relevancia de los intereses, necesidades y posibilidades del aprendizaje del estudiante, a través de actitudes y aptitudes de comunicación didáctica, formación abierta y flexible que estimule la crítica, la información y una cultura de constante transformación. En este marco, se llevó a cabo El proyecto titulado "Robot Luchador" con alumnos de tercer semestre del NMS, siguiendo cada una de las etapas que establecer el proyecto de aula, al término de dicha experiencia se identificaron las habilidades cognitivas que el alumno desarrolla cuando ha tenido la vivencia de resolver un problema en un proyecto.

La experiencia educativa se llevó a cabo con un grupo de 42 alumnos, del nivel medio superior (C.E.C.yT. 11, "Wilfrido Massieu") que cursaban la asignatura de geometría analítica. La propuesta metodológica del Proyecto Aula permitió incorporar los aprendizajes de las asignaturas en un semestre a la solución de un problema a partir de un proyecto, aplicando estrategias didácticas en todo el proceso de enseñanza aprendizaje, contribuyendo no solamente adquirir conocimientos, sino habilidades y actitudes para desenvolverse de mejor manera no sólo en el ámbito académico, sino también en el social, conectando el aprendizaje con la realidad. El 
Proyecto Aula tuvo una duración de 5 meses iniciando en el periodo intersemestral y concluyendo al término del ciclo escolar, las etapas que constituyen el proyecto aula son las siguientes:

$1^{\circ}$ Etapa. Reunión de maestros comunes del grupo para iniciar la planeación a partir de la elaboración de una red de relaciones entre los objetivos de aprendizaje de las asignaturas.

$2^{\circ}$ Etapa. Reunión de maestros, tutores y estudiantes para definir el tema central del proyecto, hipótesis o conjetura provisional, la intervención de cada asignatura, los productos esperados y las formas de evaluar el proyecto.

$3^{\circ}$ Etapa. Cada docente debe elaborar su plan de curso/proyecto, cubriéndolos resultados de aprendizaje de la asignatura y sus aportes al proyecto.

$4^{\circ}$ Etapa Reuniones para enlazar las actividades entre las asignaturas, calendarizar las sesiones para el control y evaluación participativa así como para elaborar el anteproyecto e informar el área de coordinación.

$5^{\circ}$ Etapa. Esta etapa se refiere al desarrollo de las actividades planeadas en cada asignatura que revisa el cumplimiento de los aprendizajes esperados, los avances y resultados planeados para todo el proyecto.

$6^{\circ}$ Etapa. Corresponde a la conformación del producto final, difusión de los resultados y evaluación del proyecto.

Bajo este esquema se llevó a cabo el proyecto denominado el Robot Luchador, cuyo objetivo fue elaboración y conexión de circuitos para el sistema de un Robot Luchador, desarrollando en el estudiante habilidades que le permitan construir un robot luchador, utilizando los principios básicos de robótica que coadyuven a un proceso industrial y cuyo producto integrador fue la construcción de un prototipo (robot luchador) elaborado con una placa fenólica, demostrando la habilidad manual y uso de los instrumentos de medición. Para lo cual se construyo la matriz de competencias entre las asignaturas que participaron, lo cual se presenta en la Tabla 1.

Tabla. 1: Esquema general de la participación de las asignaturas en el proyecto aula.

\begin{tabular}{|l|l|}
\hline Asignatura & Competencias \\
\hline $\begin{array}{l}\text { Geometría } \\
\text { Analítica }\end{array}$ & $\begin{array}{l}\text { Resuelve problemas de lugares geométricos, en particular de la línea recta empleando } \\
\text { las propiedades del plano cartesiano en situaciones académicas y sociales. } \\
\text { Resuelve problemas que involucren ecuaciones de segundo grado y su representación } \\
\text { gráfica, mediante la identificación de los elementos específicos de las cónicas; } \\
\text { particularmente la circunferencia, en situaciones académicas y sociales. }\end{array}$ \\
\hline Física I & $\begin{array}{l}\text { Aplicación del método científico. } \\
\text { Aplicar la suma de vectores por método grafico, paralelo triangular y poligonal. } \\
\text { acelerado, caída libre a problemas. }\end{array}$ \\
\hline Química I & $\begin{array}{l}\text { Elaboración y conexión de circuitos para el sistema de un Robot Luchador. } \\
\text { Investigar sobre propiedades y características de los materiales del robot luchador. }\end{array}$ \\
\hline Inglés III & $\begin{array}{l}\text { Manejo del vocabulario, expresiones y frases cotidianas en los tiempos presente perfecto } \\
\text { y pasado. }\end{array}$ \\
\hline $\begin{array}{l}\text { Comunicación } \\
\text { Científica }\end{array}$ & $\begin{array}{l}\text { Desarrolla el manejo de vocabulario y habilidades expositivas en expresiones científicas } \\
\text { de los conocimientos vistos en el proyecto }\end{array}$ \\
\hline $\begin{array}{l}\text { Medición e } \\
\text { Instrumentación } \\
\text { Electrónica }\end{array}$ & $\begin{array}{l}\text { Realiza conexiones de componentes eléctricos en la obtención de los valores de las } \\
\text { variables presentes en un circuito de corriente directa y corriente alterna. } \\
\text { Maneja el osciloscopio y generado de señales para desplegar la amplitud de una señal } \\
\text { en función del tiempo. }\end{array}$ \\
\hline $\begin{array}{l}\text { Electrotecnia de } \\
\text { Corriente } \\
\text { Directa }\end{array}$ & $\begin{array}{l}\text { Genera electricidad aplicando los principios físicos de la materia y la energía en } \\
\text { procesos de transformación en contextos industriales. } \\
\text { Resuelve problemas de circuitos de corriente continua y corriente alterna en función de } \\
\text { las leyes y principios que las rigen. }\end{array}$ \\
\hline $\begin{array}{l}\text { Técnicas de } \\
\text { Supervisión en } \\
\text { la Telecomu- } \\
\text { nicaciones }\end{array}$ & \begin{tabular}{l} 
Proceso de producción tipo de material a utilizar y maquinaria y equipo utilizado. \\
\hline
\end{tabular}
\end{tabular}


Cada una de las asignaturas participó en la elaboración del anteproyecto e informaron al área de coordinación que fue Medición e Instrumentación Electrónica, los avances en cada una de las etapas en particular la etapa 5. Bajo este marco de trabajo se identificaron y analizaron las habilidades cognitivas que el alumno de tercer semestre desarrolla en la asignatura de geometría analítica para la construcción del Robot Luchador. Esta investigación, se ubica en un paradigma de investigación cualitativo. Las ideas desarrolladas en los referentes teóricos, sirvieron como ejes para incorporar el proyecto de aula y se llevó a cabo un estudio de caso que permitió reconocer el progreso del grupo. Los instrumentos utilizados para la recolección de datos durante la investigación fueron: i) Reportes escritos elaborados en forma individual; ii) Reportes escritos elaborados por cada pareja de estudiantes; iii) Grabaciones en audio del trabajo de los estudiantes; y iv) Reportes elaborados por el profesor-investigador.

\section{ANÁLISIS}

Las condiciones cognitivas, que menciona Duval (2002) referentes al empleo de al menos dos representaciones, es decir la coordinación de varios sistemas semióticos, para ser integrada a la Arquitectura Cognitiva del estudiante, así como nuevos sistemas de representación, para su coordinación. Ello implica la necesidad de considerar las actividades cognitivas de tratamiento y Conversión, para lograr la aprehensión del objeto, y por consecuencia el fortalecimiento de la Arquitectura Cognitiva, lo cual contribuye a crear y desarrollar habilidades en el estudiante para enfrentar nuevos retos en su formación. Por su parte, Parnafes y DiSessa (2004) indican que la reflexión de los estudiantes esta ligada a la representación y al contexto que emplean, indicando que cada representación resalta un aspecto del concepto y que cuando los estudiantes emplean varias representaciones desarrollan un entendimiento más flexible del concepto. Parnafes y DiSessa se basaron en la nación del efecto representacional para examinar la idea de que particularmente diferentes representaciones apoyan y fortalecen diferentes procesos cognitiva, en particular, los distintos tipos de razonamiento.

Bajo esta visión se analizaron y discutieron la participación de los estudiantes en el proyecto de aula, aludiendo la evaluación de las capacidades cognitivas de los estudiantes, para identificar los elementos cognitivos conceptuales que atañen a los contenidos incorporados al trabajo de modelar, comprensión de los sistemas de representaciones y conceptualización, que abordan el reconocimiento de conceptos e interpretación del concepto. El análisis de la experiencia se estructuro en tres fases: Fase I. Introducción a la problemática y las primeras aproximaciones del anteproyecto, Fase II. Identificación y tratamiento de las representaciones empleadas, Fase III. Exposición de sus resultados y la comunicación de los mismos al grupo. En la Tabla 2 se exponen las diferentes aproximaciones que los estudiantes desarrollaron para abordar el proyecto de aula desde la asignatura de Geometría Analítica.

Tabla 2: Análisis y discusión sobre los hallazgos identificados durante el proyecto; Fase I

\begin{tabular}{|l|l|}
\hline \multicolumn{2}{|l|}{ Fase I } \\
\hline Habilidades Desarrolladas & Descripción \\
\hline Interpreta el Proyecto & Idea vaga del objetivo del proyecto. \\
\hline $\begin{array}{l}\text { Identifica la participación de las } \\
\text { Unidades de Aprendizaje involucradas } \\
\text { en la construcción del Robot luchador }\end{array}$ & $\begin{array}{l}\text { Esbozo general de la participación de las asignaturas, } \\
\text { participación de los profesores de cada una de las asignaturas. }\end{array}$ \\
\hline $\begin{array}{l}\text { Identifica la participación de la Unidad } \\
\text { de Aprendizaje de Geometría Analítica. }\end{array}$ & $\begin{array}{l}\text { Idea vaga de la participación de la asignatura de geometría } \\
\text { analítica en el proyecto. }\end{array}$ \\
\hline $\begin{array}{l}\text { Organiza y explicita diferentes } \\
\text { procedimientos para enfrentar los datos } \\
\text { obtenidos }\end{array}$ & $\begin{array}{l}\text { Idea aproximada del comportamiento de los datos obtenidos } \\
\text { para ubicar los puntos en donde se encuentran cada uno de los } \\
\text { robots luchadores destinados a combatir. }\end{array}$ \\
\hline $\begin{array}{l}\text { Reconoce la necesidad de establecer } \\
\text { un modelo para los datos obtenidos }\end{array}$ & $\begin{array}{l}\text { Establece diferentes acercamientos para establecer relaciones e } \\
\text { identificar las variables que participan. }\end{array}$ \\
\hline
\end{tabular}


Tabla 2: (continuación)

Organiza e interpreta la información recopilada

Establece relaciones para identificar el comportamiento de los datos.

Discusión; Los alumnos muestran un reconocimiento global para el diseño del robot, y ubican en el proyecto la participación de la asignatura de geometría analítica, reconocen que la representación gráfica podría contribuir al análisis de los desplazamientos de los robots, que permiten delimitar alcances. Se destaca la organización en la planificación y diseño del robot luchador, ya que el grupo plantea diversas presentaciones mostrando la información recopilada y muestran los diversos estudios relacionados con la asignatura de medición e instrumentación electrónica, para determinar la trayectoria que seguirá el robot. Aunque no se tiene una idea clara del tratamiento de la información.

Tabla 3: Análisis y discusión sobre los hallazgos identificados durante el proyecto; Fase II

\begin{tabular}{|l|l|}
\hline Fase II & \multicolumn{2}{|l|}{$\begin{array}{l}\text { Emplea y reconoce los sistemas de } \\
\text { representación. }\end{array}$} & $\begin{array}{l}\text { Durante la experiencia los estudiantes emplearon las } \\
\text { representaciones; gráfica, simbólica, pictográfica, concretas y } \\
\text { numérica. }\end{array}$ \\
\hline $\begin{array}{l}\text { Identifica el contenido de las } \\
\text { representaciones empleadas }\end{array}$ & $\begin{array}{l}\text { Durante la experiencia los estudiantes emplearon diferentes } \\
\text { diagramas para el diseño del robot y su transferencia a los } \\
\text { circuitos permitió la construcción del robot. Por otro lado el trazo } \\
\text { de diferentes gráficas para el comportamiento de los robots, } \\
\text { presentaron dificultades debido a las escales empleadas. }\end{array}$ \\
\hline $\begin{array}{l}\text { Reconoce el tipo de expresión que } \\
\text { permita la modelización de la situación. }\end{array}$ & $\begin{array}{l}\text { Se presentó rigidez en el uso de la representación algebraica } \\
\text { para la construcción del modelo inicial. Mediante las preguntas } \\
\text { planteadas por el docente los estudiantes pasaron a otro nivel en } \\
\text { donde identificaron nueva información, lo que llevó a revisar el } \\
\text { problema planteado originalmente. }\end{array}$ \\
\hline $\begin{array}{l}\text { Aplica diferentes procedimientos en la } \\
\text { representación numérica para identificar } \\
\text { su contenido. }\end{array}$ & $\begin{array}{l}\text { Se presentaron dificultades en la lectura de las gráficas } \\
\text { contempladas en algunas de las tareas }\end{array}$ \\
\hline $\begin{array}{l}\text { Interpreta la información en las } \\
\text { representaciones numérica y algebraica } \\
\text { de la expresión identificada }\end{array}$ & $\begin{array}{l}\text { Se presentaron diferentes aproximaciones para la identificar los } \\
\text { parámetros de las cónicas, específicamente la circunferencia. }\end{array}$ \\
\hline $\begin{array}{l}\text { Relaciona el comportamiento de los } \\
\text { datos con la gráfica }\end{array}$ & $\begin{array}{l}\text { Particularmente se trabajó la circunferencia para el estudio del } \\
\text { Robot Luchador }\end{array}$ \\
\hline
\end{tabular}

Discusión: En esta fase los estudiantes hacen uso de múltiples representaciones, e identifican las distancias entre puntos existentes en la arena donde se efectuará la lucha de los robots, también denominado tatami o dohyo. En base a éste análisis los alumnos emplearon el tema de distancia entre dos puntos, para el diámetro que debe medir el dohyo, obteniendo el área de la arena de peleas.

El tema relacionado fue la circunferencia para la construcción de la arena donde se llevó a cabo las peleas de los robots.

El uso de múltiples representaciones como son: gráfica, numérica, algebraica, pictográfica y concreto, permitió examinar el proyecto de diversos ángulos, proporcionando ingredientes fundamentales para que el estudiante analice cualidades matemáticas relacionadas con el área de la arena de peleas, por ejemplo, mientras la obtención de diversas coordenadas en la tabla numérica representa un conjunto de puntos discretos, la gráfica dio cuenta de una visión continua y visual del fenómeno, permitiendo el desarrollo de estrategias para formular conjeturas y en algún momento diseñar y proponer argumentos (habilidad cognitivo- lingüística) sólidos en el proyecto, como es el cambio de condiciones iniciales o bien extender exponer diferentes sugerencias para la mejora del Robot Luchador.

Los diferentes tratamientos que se desarrollaron fueron del tipo cualitativo y cuantitativo, en algunos casos se establecieron conexiones no solamente dentro de la disciplina sino también con las demás asignaturas que participaron en el proyecto, lo que contribuyo a que el alumno formulara conjeturas las cuales se argumentaron para establecer posibles generalizaciones. Dando muestra del fortalecimiento de las habilidades cognitivas: interpretar, analizar y deducir. 
Tabla 4: Análisis y discusión sobre los hallazgos identificados durante el proyecto; Fase III

Fase III

Expresa conceptos en distintas representaciones (gráfica, numérica, verbal, algebraica...)

Elabora ejemplos y contraejemplo

Aplica correctamente los conceptos en el proyecto.

Comunica los resultados obtenidos en el análisis y explicita el proceso seguido en le trabajo con las gráficas y datos.
Confusiones iniciales en el tratamiento cualitativo y cuantitativo en la representación gráfica y algebraica para interpretar su contenido.

El alumno en esta etapa identifica y analiza a través de los tratamientos empleados el concepto de variación y el impacto que tiene en el desarrollo del proyecto.

Inclinación a formular ejemplos o contraejemplos que eran fáciles de identificar.

Los estudiantes establecieron las relaciones matemáticas de una situación y la conexión de la información, identificando tratamientos cualitativos y cuantitativos que permitieran establecer relaciones entre las representaciones empleadas.

Al inicio el alumno presento dificultad para comunicar ideas matemáticas, tanto en forma oral como escrita. Las preguntas planteadas no expresaban el trabajo desarrollado al interaccionar con la tarea, así mismo sucedía para enunciar un problema, a pesar de que se había entendido la esencia de la situación.

No obstante, durante el proceso los estudiantes presentaron argumentos de los conceptos empleados y procesos desarrollados, permitiendo establecer conjeturas fundamentadas para comunicarlas a un grupo inmerso en una misma problemática.

Discusión: En esta fase el estudiante selecciono y manejo la información, pues durante el proceso se eligieron datos relevantes. Además, muestran un desarrollo organizado al identificar el problema concreto para el estudio, así como la búsqueda de la información, y la planificación de las tareas a realizar.

La visión a futuro del grupo fue expuesta de manera clara, evidenciando la capacidad visionaria para solucionar el problema y establecer sus propuestas a trabajos futuros, además su expresión oral dejó constancia de que se encontraron a un nivel distinto al del inicio del proyecto, desarrollando sus habilidades comunicativas.

De manera general, el proyecto proporcionó una plataforma sólida para realizar exploraciones, reconocer conjeturas y eventualmente proporcionar argumentos que las soporten, fortaleciendo la visualización, el reconocimiento y la argumentación que son procesos fundamentales del quehacer de la matemática pero que contribuyen fuertemente en el desarrollo de las disciplinas que participan.

\section{CONCLUSIONES}

1.- La educación en el nivel medio superior debe encaminar el aprendizaje del futuro profesional hacia el desarrollo de habilidades que integren todas las gamas del conocimiento, proporcionado al alumno la oportunidad de realizar prácticas que ligan los conceptos teóricos profesional en el contexto que los rodea.

2.- El proyecto de aula es una metodología que permite fortalecer las habilidades cognitiva: interpretar, analizar sintetizar, deducir y razonar, ya que proporciona el espacio idóneo para que el estudiante discuta un problema real, teniendo la oportunidad de llevar cabo exploraciones, reconocimientos, y conjeturas que serán argumentadas y validadas en reuniones consensuadas.

3.- Es notorio el avance de un número de alumnos en la adquisición de habilidades comunicativas (hablar en público y defender sus opiniones), empleando la metodología del proyecto de aula, no obstante se presentaron dificultades en la expresión de conceptos en distintos lenguajes.

4.- La Interpretación Global es una vía que permite identificar información relevante tanto en la representación gráfica, pictográfica, numérica y algebraica, para explorar de manera conjunta su trascendencia entre las distintas representaciones. En este sentido, las representaciones aportan información relevante que beneficia la identificación de información, para identificar los valores 
numéricos de los coeficientes que integran a la expresión algebraica, lo cual fortalece las habilidades cognitivas en el estudiante.

5.- Es necesario tomar conciencia de la importancia de la evaluación, que genera una reflexión enriquecedora sobre la realidad evaluativa para llegar a comprender lo que significa educar desde el enfoque por proyectos.

6.- La manera en que se organizaron las actividades en el curso, es decir, trabajo en equipo, exposiciones y discusión grupal, fueron elementos que aportaron para que el alumno pudiera exponer sus ideas y conjeturas.

\section{AGRADECIMIENTOS}

Las autoras agradecen el patrocinio otorgado por la Comisión y Fomento a las Actividades Académicas [COFAA-IPN] para realizar y presentar este artículo. Las investigaciones con números de registro 20100459 y 20100678 han sido apoyadas por la SIP del IPN.

\section{REFERENCIAS}

Abrantes, $\mathrm{P}, \mathrm{O}$ trabalho de projecto e a relaçao dos alunos com a matemática a expreriência do projecto MAT789. Tesis doctoral no publicada, Universidad de Lisboa, Portugal (1994).

Aravena, M, Evaluación de proyectos para un curso de álgebra universitaria. Un estudio basado en la modelización polinómica. Tesis doctorado, Universidad de Barcelona, España (2001).

Ausubel, D.; Novak, J. y Hanesian, H. Psicología Educativa, Un punto de vista cognoscitivo. Trillas Editores, D.F., México (1983).

Benítez, A. Estudio de la Primera Representación Gráfica de las Ecuaciones Algebraicas en Contexto, Innovación Educativa: 9 (1), 41-50 (2009).

Doveston, M. y Keenaghan, M., Growing Talent for Inclusion: using an appreciative inquiry approach into investigating classroom dynamics, Journal of Research in Special Educational Needs, 6(3), 153-165 (2006).

Duval, R. Representation, vision and visualization: cognitive functions in mathematical thinking. The XXIV Annual Conference of North American Chapter of international Group for the Psychology of Mathematics Education, 311-335, Georgia, USA 23 a 26 de Octubre, (2002).

Gómez, J. La Modelización Matemática. Una herramienta válida en la enseñanza de las Matemáticas Universitarias. Sumas, 42, 37-35 (2003).

Goldin, G. y Kaput, J. A joint perspective on the idea of representation in learning and doing mathematics. Theories of mathematical learning, 987-439, Hillsdale, NJ:Erlbaum (1996).

Hendrickson, K.A, The "Farmer Dan" Project: Implementation of Japanase Lesson Study in Rural Mathematics Classrooms. The $32^{\text {nd }}$ Annual Conference of North American Chapter of International Group for the Psychology of Mathematics Education, 1398-1401, Columbus, USA 28 a 31de Octubre (2010).

Materiales para la Reforma. Un Nuevo Modelo Educativo para el IPN. Instituto Politécnico Nacional. México 2004.

Janvier, C. Problems of representation in the teaching and learning of mathematics. Hillsdale: Lawrence Earlbaum New Jersey, USA (1987). 
Kaput, J. Notations and representations as mediators of constructive processes, Radical constructivism in mathematics education, 53-74, Dordrecht: Kluwer Academic Publisher USA (1991).

Koretsky, M.D., Amatore, D., Barnes, C. y Kimura, S.; Enhancement of Student Learning in Experimental Design Using a Virtual Laboratory. 4448426 abstract, Education, IEEE Transactions, 51(1), 76 - 85 (2008).

Lesh R. y Lehrer, R. Models and Modeling Perspective on the Development of Students and Teachers. Mathematical Thinking and Learning, 5, 2-3, 109-129 (2003).

Morrison, D., Using Activity Theory to Design Constructivist Online Learning Environments for Higher Order Thinking: A Retrospective Analysis, Canadian Journal of Learning and Technology, 29(3) 4-9 (2003).

Lesh R. y Lehrer, R. Models and Modeling Perspective on the Development of Students and Teachers. Mathematical Thinking and Learning, 5, 2-3, 109-129 (2003).

Niss, M. Aim and of applications and modeling in mathematics curricula. Applications and modeling in learning and teaching mathematics 22-32 UK, Chichester: Ellis Horwood (1989).

Parnafes, O. y diSessa, A. Relations between patterns of reasoning and computational representations. International Journal of Computers for the Mathematics Learning: 9, 251-280. (2004).

Ricks, T.E. Juxtaposing Chinese and American Mathematics Education Commitments. The $32^{\text {nd }}$ Annual Conference of North American Chapter of International Group for the Psychology of Mathematics Education, 1398-1401, Columbus, USA 28 a 31 de Octubre (2010).

Thomas, J.W. Project based learning overview. Novato, CA: Buck Institute for Education, (1998). 